\title{
Effect of Using a Counter-balanced Smith Machine on Performance Measurements for Concentric-Only Bench Press Throws
}

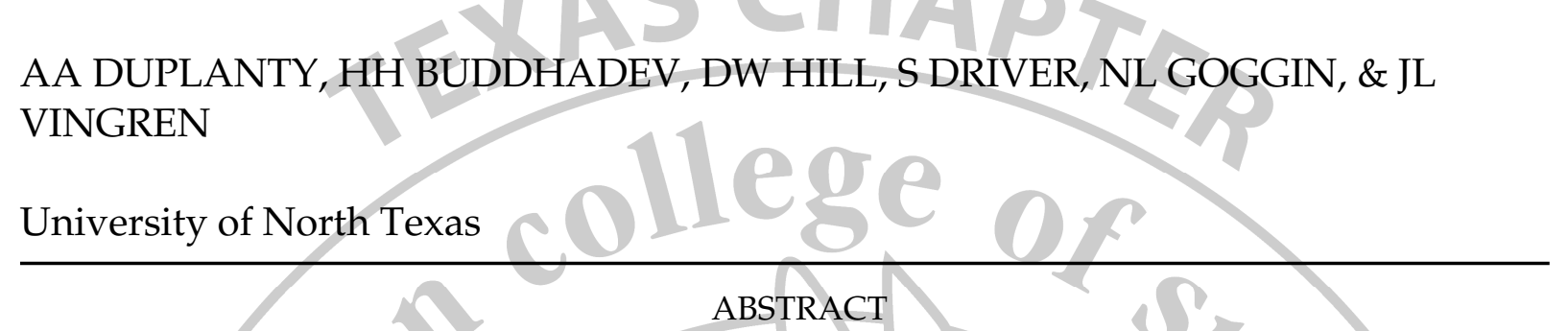

Bench press throws using a Smith machine are often used for assessment and training of upper body power. Concentric only bench press throws (CON-BT) provide important information on an individual's ability to produce force from a static start. Smith machines often utilize a counter-balance weight system to reduce the net load on the barbell; however, it is not known how counter-balance weight affects measurements of performance during a CON-BT. PURPOSE: To examine the effect of a counter-balance weight on CON-BT performance measurements. METHODS: 24 men (age: $23 \pm 3$ years, height: $179 \pm 6$ $\mathrm{cm}$, mass: $91 \pm 17 \mathrm{~kg}$, bench press 1-repetition maximum [1RM]: $107 \pm 18 \mathrm{~kg}$ ) performed 2 sets of 2 repetitions of CON-BT at 30\% of their $1 \mathrm{RM}$ using a no counter-balanced (NCB) and a counter-balanced (CB) Smith machine. Total duration, peak power, peak force, and peak velocity were measured using a linear accelerometer attached to the barbell; peak ground reaction force (GRF) was measured using a force plate. For each condition, data from the repetition with the highest peak power was used for further analyses. Peak EMG was measured for the right pectoral, deltoid and triceps muscles and normalized using peak EMG in the 1RM. RESULTS: Measurements for peak barbell power (NCB: $1169 \pm 260 \mathrm{~W}$, CB: $938 \pm 262 \mathrm{~W})$ and force (NCB: $695 \pm 129 \mathrm{~N}, \mathrm{CB}: 577 \pm 134 \mathrm{~N})$ were significantly greater $(p<0.05)$ for NCB compared to $\mathrm{CB}$. The total duration of CON-BT was shorter for NCB $(0.62 \pm 0.41 \mathrm{~s})$ compared to CB $(0.78$ $\pm 0.50 \mathrm{~s})$. The peak GRF showed a trend $(p<0.10)$ for being lower for NCB $(884 \pm 213 \mathrm{~N}]$ compared to CB [912 \pm 190 N). Peak EMG and peak velocity were unaffected by the use of counter-balance weight. CONCLUSION: The use of a CB Smith machine reduced barbell performance measurements (peak power and peak force) but increased the peak GRF during a CON-BT. A counter-balance weight becomes ineffective and the net external load increases during the CON-BT when the barbell accelerates faster than the gravitational constant pulls on the counter weight, thus explaining the lower performance measurements found for CB. 